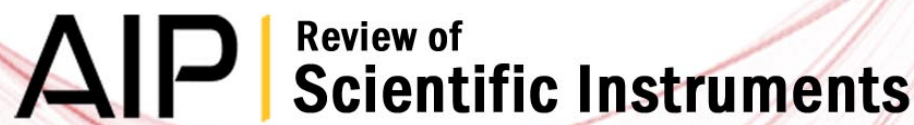

A direct digital synthesis chirped pulse Fourier transform microwave spectrometer

Ian A. Finneran, Daniel B. Holland, P. Brandon Carroll, and Geoffrey A. Blake

Citation: Rev. Sci. Instrum. 84, 083104 (2013); doi: 10.1063/1.4818137

View online: http://dx.doi.org/10.1063/1.4818137

View Table of Contents: http://rsi.aip.org/resource/1/RSINAK/v84/i8

Published by the AIP Publishing LLC.

\section{Additional information on Rev. Sci. Instrum.}

Journal Homepage: http://rsi.aip.org

Journal Information: http://rsi.aip.org/about/about_the_journal

Top downloads: http://rsi.aip.org/features/most_downloaded

Information for Authors: http://rsi.aip.org/authors

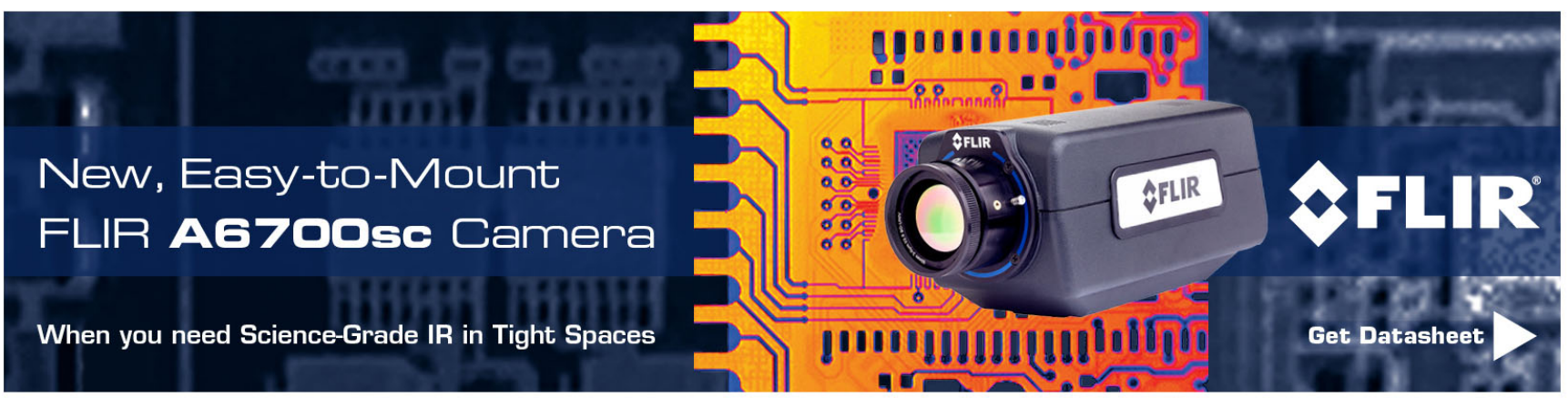




\title{
A direct digital synthesis chirped pulse Fourier transform microwave spectrometer
}

\author{
Ian A. Finneran, ${ }^{1}$ Daniel B. Holland, ${ }^{1}$ P. Brandon Carroll, ${ }^{1}$ and Geoffrey A. Blake ${ }^{2}$ \\ ${ }^{1}$ Department of Chemistry, California Institute of Technology, Pasadena, California 91125, USA \\ ${ }^{2}$ Divisions of Geological and Planetary Sciences and Chemistry and Chemical Engineering, California \\ Institute of Technology, Pasadena, California 91125, USA
}

(Received 4 June 2013; accepted 27 July 2013; published online 15 August 2013)

\begin{abstract}
Chirped pulse Fourier transform microwave (CP-FTMW) spectrometers have become the instrument of choice for acquiring rotational spectra, due to their high sensitivity, fast acquisition rate, and large bandwidth. Here we present the design and capabilities of a recently constructed CP-FTMW spectrometer using direct digital synthesis (DDS) as a new method for chirped pulse generation, through both a suite of extensive microwave characterizations and deep averaging of the $10-14 \mathrm{GHz}$ spectrum of jet-cooled acetone. The use of DDS is more suited for in situ applications of CP-FTMW spectroscopy, as it reduces the size, weight, and power consumption of the chirp generation segment of the spectrometer all by more than an order of magnitude, while matching the performance of traditional designs. The performance of the instrument was further improved by the use of a high speed digitizer with dedicated signal averaging electronics, which facilitates a data acquisition rate of 2.1 kHz. @ 2013 AIP Publishing LLC. [http://dx.doi.org/10.1063/1.4818137]
\end{abstract}

\section{INTRODUCTION}

Microwave spectroscopy is an invaluable tool for studying the structure, ${ }^{1}$ dynamics, ${ }^{2}$ and even the handedness ${ }^{3}$ of gas phase species. In particular, the specificity of microwave spectroscopy has been central to the unambiguous identification of the great majority of molecules detected in the interstellar medium. ${ }^{4}$ Applications of microwave spectroscopy to problems in physical chemistry and molecular astrophysics have been greatly accelerated by developments in laboratory techniques. One of the most significant advancements in microwave spectroscopy was the invention of pulsed microwave emission techniques in the 1950s, which provided increased sensitivity over traditional absorption experiments. ${ }^{5}$ Further increases in sensitivity and resolution over small bandwidths were demonstrated in the 1970s and 1980s by the Flygare lab at the University of Illinois, using both waveguide and FabryPerot resonator spectrometers. ${ }^{6,7}$ In the mid-2000s the Pate lab at the University of Virginia developed a complementary Chirped Pulse Fourier Transform MicroWave (CP-FTMW) spectrometer, capable of measuring $11 \mathrm{GHz}$ of bandwidth in less than $10 \mu \mathrm{s} .{ }^{8}$ The bandwidth, acquisition speed, sensitivity, and relative intensity information attainable with the chirped pulse design has led to its increasingly widespread use in the molecular spectroscopy community. ${ }^{9-16}$

There are three major components of CP-FTMW spectrometers that enable the collection of broadband spectra: high sample rate digitizers for acquisition of the microwave emission, broadband high power amplifiers (based on either solid state or traveling wave tube technology) to ensure sufficient power for sample polarization, and Arbitrary Waveform Generators (AWGs) or Arbitrary Function Generators (AFGs) for producing the chirped polarization pulse. In state-of-the-art, multi-GHz bandwidth instruments each of these major components require significant power and physical space. AFGs and AWGs, in particular, are bulky and energy-consuming, since they contain a significant amount of microwave circuitry so as to enable the generation of arbitrarily complex pulse sequences. However, for most CP-FTMW experiments (with a few notable exceptions, such as 2D MWMW spectroscopy ${ }^{17}$ ) the waveform generator is only used to create pulsed linear frequency sweeps. A simpler and morecompact alternative to AWGs/AFGs is direct digital synthesis.

Direct digital synthesizers (DDS) are Nyquist devices with good frequency agility and low phase noise. Using an external sample clock and digital control word, a DDS generates a tunable digital signal with a numerically controlled oscillator, which is then converted into sinusoidal output with a digital-to-analog converter (DAC). ${ }^{18}$ Their frequency agility and low phase noise capabilities have been utilized for longer (35 $\mu \mathrm{s}$ ) broadband frequency sweeps at millimeter-wave frequencies for radar imaging applications,${ }^{19}$ as well as slow narrowband frequency sweeps $(3 \mathrm{MHz}$ ) in a millimeter-wave fast scan absorption spectrometer. ${ }^{20}$ To date, however, this technology has never been used for CP-FTMW spectroscopy.

To this end, we discuss the novel use of a DDS chip to generate short $(\sim 1 \mu \mathrm{s})$, broadband $(1.9 \mathrm{GHz})$ linear frequency sweeps for CP-FTMW spectroscopy. ${ }^{21} \mathrm{~A}$ second PLL board functioning as the local oscillator (LO) source further reduces the weight and size of the spectrometer. The total power requirement of the DDS and PLL boards is only $3 \mathrm{~W}$, considerably less than the power draw of an AWG, 100$500 \mathrm{~W}$. The DDS and PLL boards are also much smaller and lighter $\left(1400 \mathrm{~cm}^{3}, 240 \mathrm{~g}\right)$ than an AWG $\left(42000 \mathrm{~cm}^{3}, 14.1 \mathrm{~kg}\right.$ for the $4.2 \mathrm{GS} / \mathrm{s}$ Tektronix AWG710B), making it possible to use CP-FTMW spectrometers in many applications that were previously not feasible due to size, weight, or power restrictions. For this paper all of the components were purchased and soldered into separate non-optimal evaluation boards for proof-of-principle experiments. However, the chips (DDS, PLL) used in these boards are sold individually, and could be 
combined into a single compact circuit board that has been optimized for the needs of the CP-FTMW spectrometer, further reducing the weight, size, and power required. The current evaluation board spectrometer and future optimized circuit designs will make the CP-FTMW technique more suitable for in situ applications.

\section{EXPERIMENTAL}

Many variations on the original CP-FTMW microwave spectrometer have been described in the literature, ${ }^{9-16}$ but they are all based on the same basic theory of operation. A phase-stable broadband microwave pulse from an AWG is amplified and broadcast into a gas phase molecular sample, either in a static cell ${ }^{10}$ or in a molecular beam expansion. ${ }^{8}$ The coherent microwave pulse builds up a macroscopic polarization in the gas, as the molecular dipoles rotate in phase with the microwave pulse. Before the dipoles dephase, the microwave pulse ends and the molecules continue to rotate in phase, emitting a free induction decay (FID). Because the angular momentum of the molecules is quantized, they only rotate at specific frequencies that are dependent on their moments of inertia. The FID is detected in the time domain with a digitizer and Fourier transformed to recover the frequency domain spectrum. Since the frequencies that are probed with the microwave pulse are often higher than the bandwidth of the AWG and the digitizer, the chirped pulse and FID are typically heterodyned with a higher frequency signal from a microwave synthesizer or phase-locked dielectric resonant oscillator.

Our spectrometer differs from previous designs in three ways: the AWG has been replaced by a DDS board, the microwave synthesizer has been replaced by a PLL synthesizer board, and the chirp is broadcast into a coaxial expansion with one horn and microwave foam behind the pulsed valve. To utilize the full $2 \mathrm{GHz}$ Nyquist bandwidth of the digitizer, we have also synchronized the DDS board and the digitizer to the same $4 \mathrm{GHz}$ sample clock.

\section{A. Microwave circuit}

A schematic of our spectrometer is shown in Figure 1. The DDS evaluation board (Analog Devices 9914) generates a $1.9 \mathrm{GHz}$ linear frequency sweep chirp in $1.2 \mu \mathrm{s}$. The chirp is low-pass filtered (Minicircuits, VLP-24) and then upconverted by mixing with the doubled (Marki ADA-0410P) output of a PLL evaluation board (Analog Devices ADF4158) in a broadband mixer (Marki M1R-0726L), to reach a final dual sideband bandwidth of $3.9 \mathrm{GHz}$ centered at $11.9 \mathrm{GHz}$. A gap exists between 11.85 and $11.95 \mathrm{GHz}$. After upconversion, the chirp is amplified by a $40 \mathrm{~W}$ solid state amplifier (Microsemi C0618-43-T680), passed through a circulator (Teledyne C-12S63T-1), and broadcast into a vacuum chamber $\left(\sim 10^{-6}\right.$ Torr) through a microwave horn (Narda model 640 ). The gas sample is supersonically expanded coaxially (with respect to the microwave pulse) through a pulsed nozzle (General Valve Series 9) positioned at the center of a section of microwave foam (Emerson and Cuming HR-25). Molec-

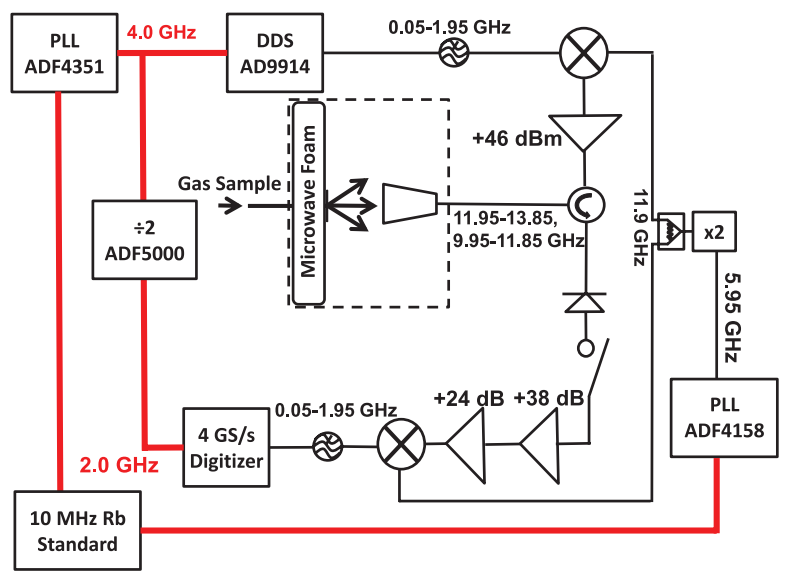

FIG. 1. The microwave circuit of the DDS-based chirped pulse spectrometer. Microwave reference connections are highlighted for clarity. See text for design details.

ular sample is fed into the pulsed valve by sending argon through a gas bubbler containing the sample. While the microwave radiation is passing through the circulator, an open SPST switch (Narda S213D) prevents the pulse from damaging the detection path of the circuit, while a diode limiter (Aeroflex ACLM-4537C361K) prevents damage to the switch. The timing of the switch, the solid state amplifier, and the start of the DDS chirp are controlled by a digital pulse delay generator (SRS DG-535). After excitation, the microwave horn collects the molecular emission and directs it to the detection portion of the circuit through the circulator. The emission is amplified by a high-gain, low noise amplifier (Miteq AMF-5F-08001800-14-10P), downconverted by a second mixer (Marki M1R-0726L), low-pass filtered (Minicircuits, VLP-24), and sampled by a 4 GS/s digitizer (Agilent U1084A).

For the AWG tests we exchanged the DDS board with the AWG used in the original CP-FTMW paper ${ }^{8}$ (Tektronix AWG7102, $10 \mathrm{GS} / \mathrm{s}$, on loan from the University of Virginia). The local oscillator (LO) PLL board (ADF4158) and doubler were also replaced with a microwave synthesizer (Hittite HMC 2100) to match the conventional CP-FTMW design. ${ }^{8}$

\section{B. Instrument triggering}

A schematic of the instrument triggering is shown in Figure 2. A critical design feature is the trigger signal sent directly from the DDS to the digitizer (trace 4 in Figure 2). When triggered, the DDS will output the chirped pulse with a random delay of up to one clock cycle, making trace 3 unusable for digitizer triggering. However, a digital high/low pin output on the DDS board does give the required precision timing for averaging waveforms on the digitizer. ${ }^{22}$

The Agilent U1084A digitizer is capable of averaging at extremely high repetition rates using the onboard FPGA (field-programmable gate array); the only limiting factor for the repetition rate is the re-arm time of $2.7 \mu$ s between consecutive waveforms. Consequently, the maximum repetition rate for a $9 \mu$ s FID collection is $85 \mathrm{kHz}$, which could be realized in a continuous expansion or static cell experiment. 


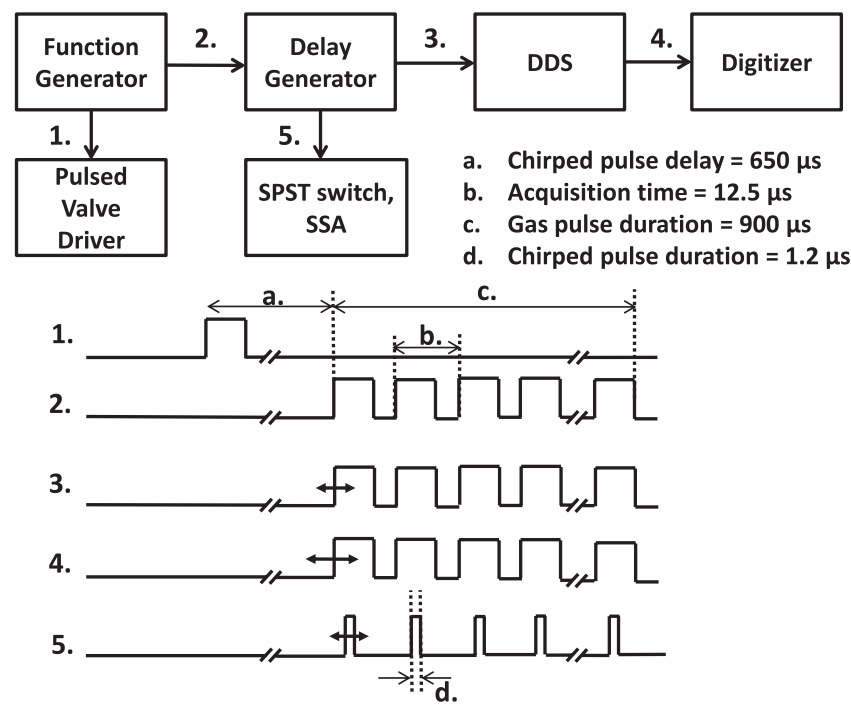

FIG. 2. The pulse timing used for collecting multiple FIDs per gas pulse. See text for design details.

Thus, our FID averaging throughput is limited by the maximum pulsed valve rate that is sustainable with our vacuum pumps. In previous CP-FTMW spectrometers multiple FIDs have been collected for each gas pulse, by outputting multiple chirped pulses from the AWG per trigger pulse. ${ }^{8}$ The DDS board, however, generates only one chirped pulse per trigger event. To circumvent this problem we triggered the delay generator with seventy $12.5 \mu$ s square pulses from the output of a function generator (SRS DS345) that were delayed by 600 $\mu$ s from the start of the synchronization signal (Figure 2). The synchronization signal was sent to the pulsed valve so that 70 chirped pulses were triggered per gas pulse. The pulsed valve was run at a repetition rate of $30 \mathrm{~Hz}$, so that the repetition rate for the $9 \mu$ s FID collection was effectively $2.1 \mathrm{kHz}$.

\section{Sample interaction region}

We were able to further improve the performance of the instrument by using a coaxial molecular beam orientation. A similar CP-FTMW spectrometer with one horn and one focusing mirror has also been demonstrated in perpendicular ${ }^{23}$ and coaxial $^{24}$ arrangements. The setup presented here is similar to the last setup, except the microwave mirror has been replaced by microwave foam so that the backwards going emission is detected (Figure 1). This setup is more appropriate for coaxial broadband measurements, since the cavity $Q$ is negligible. For a single nozzle setup, the overlap between the microwave radiation and the expansion is more optimal in the coaxial arrangement leading to a longer transit time for FID collection and thus a higher signal-to-noise ratio and higher spectral resolution.

\section{RESULTS}

To evaluate the performance of DDS-based CP-FTMW spectrometers we measured the output of the DDS board individually (with the $4 \mathrm{GS} / \mathrm{s}$ digitizer) and as part of the total microwave circuit. As an isolated component, we compared the triggering, bandwidth, and phase stability of the DDS board to the AWG. After demonstrating that the board was capable of generating a triggered, broadband, phase-stable chirped pulse, we connected it to a full CP-FTMW circuit and compared the phase stability and signal-to-noise of the new DDS/PLL-based instrument with that of the AWG/microwave synthesizer-based instrument for the FID of a transition in acetone. Phase stability of the FID is extremely important, since many data acquisition cycles must be averaged to obtain reasonable signal-to-noise ratios. Finally, we collected the full $3.8 \mathrm{GHz}$ A/D bandwidth-limited spectrum of acetone with deep averaging and compared the results with predicted spectra from the literature, to demonstrate the dynamic range and relative intensity performance of the instrument.

\section{A. Phase stability and bandwidth of the chirped pulse}

The two main sources of phase jitter in the DDS/digitizer combination are changes in the phase of the chirped pulse output of the DDS and trigger jitter between the DDS and digitizer. Regarding the first issue, the particular DDS board (AD9914) shifts the phase of the chirp output from pulse to pulse. We took advantage of the phase-reset feature by setting the "Autoclear phase accumulator" bit in the DDS chip, so that the phase was the same for every chirped pulse triggered by the delay generator. ${ }^{18}$

Trigger jitter acts as a low pass filter in the frequency domain, limiting the bandwidth of the spectrometer when multiple time domain waveforms are co-added. We minimized the trigger jitter by synchronizing the DDS and digitizer with the same external sample clock, the $4 \mathrm{GHz}$ output of the ADF4351 PLL. The maximum external clock frequency for the digitizer is $2 \mathrm{GHz}$, so we divide the $4 \mathrm{GHz}$ DDS clock by two (Analog Devices ADF5000) before it passes into the digitizer. The $2 \mathrm{GHz}$ bandwidth chirped pulse output of the DDS and the AWG are shown in Figure 3. After averaging both
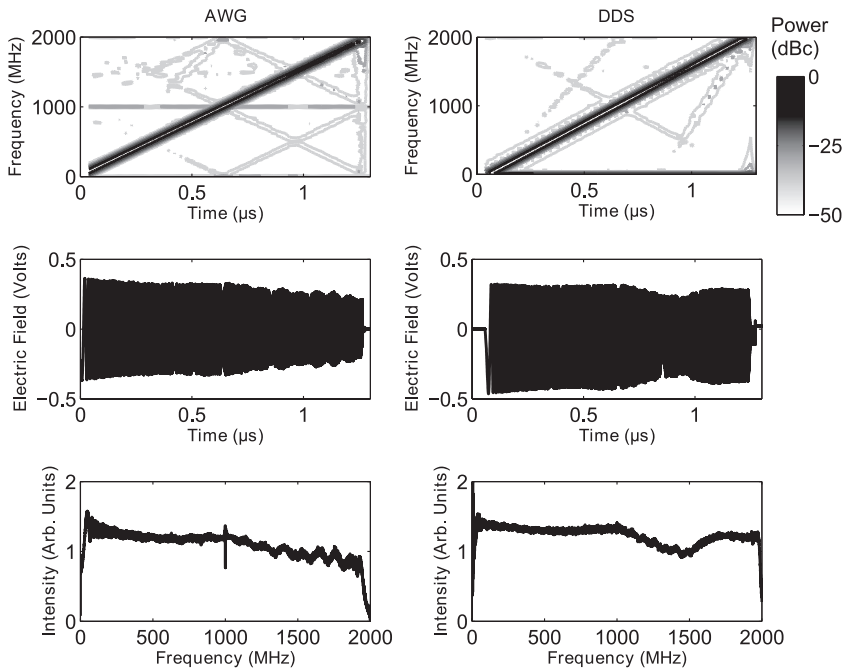

FIG. 3. A comparison between the filtered chirped pulse outputs of an AWG (left) and a DDS (right) after 100000 averages. The FFT of the waveforms are very similar, except for a small dip in the intensity of the DDS at $1.4 \mathrm{GHz}$. A spectrogram of the chirped pulses (top) indicates that the AWG and DDS have similar harmonic distortion in their output. The off diagonal content in the spectrogram is down by $30 \mathrm{~dB}$ from the main frequency sweep in both the DDS and the AWG. 


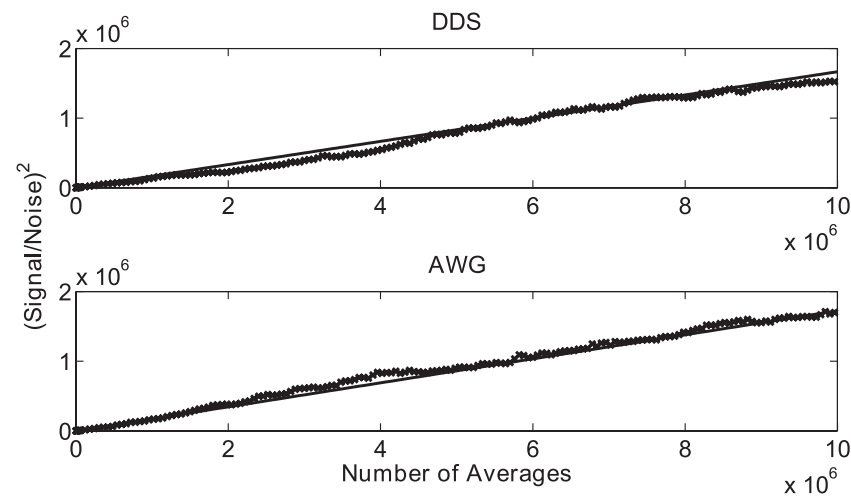

FIG. 4. The phase stability of the DDS spectrometer was compared to that of the AWG spectrometer via emission from the $3_{21}-3_{12}$ AA transition in acetone (10762.6 MHz rest frequency, $\mathrm{LO}=11900 \mathrm{MHz}$ ). Both setups show little deviation from $\sqrt{n}$ averaging.

waveforms up to 100000 times no significant intensity modulations were observed in the averaged signal of the DDS, except a small dip at $1.4 \mathrm{GHz}$. A spectrogram of the waveforms indicated that both the DDS and the AWG have similar harmonic distortion, at approximately $-30 \mathrm{dBc}$.

For normal operation, the bandwidth of the DDS is limited by the Nyquist frequency, or $2 \mathrm{GHz}$ (using a $4 \mathrm{GHz}$ sample clock). However, an inherent feature of DDS chips is low power super-Nyquist images of the fundamental. ${ }^{18}$ These images can be filtered out of the DDS signal (as is done in this design, albeit inefficiently, as shown in Figure 5) or used to extend the bandwidth of the AD9914 beyond $2 \mathrm{GHz} .{ }^{22}$

\section{B. Phase stability and signal-to-noise of the FID}

A common-mode rejection design (Figure 1) was employed to minimize LO phase jitter by splitting (Minicircuits
ZX10-2-183-S+) the doubled PLL output to drive both the upconversion and downconversion mixers (as done in previous CP-FTMW circuits ${ }^{9}$ ). To evaluate the phase stability of the instrument 10 million FIDs from the $3_{21}-3_{12}$ AA transition in acetone (10762.6 MHz rest frequency, measured with 11900 $\mathrm{MHz}$ LO) were averaged with the DDS/PLL setup and with a conventional AWG/synthesizer circuit (Figure 4). The deviation from $\sqrt{n}$ averaging is similar in both setups, indicating that the DDS spectrometer has sufficient phase stability to be used for coherent averaging in a CP-FTMW spectrometer. The total signal-to-noise at 10 million averages differed by only $4 \%$ between the AWG and DDS circuits, insignificant compared to the inherent intensity variations in CP-FTMW spectrometers. ${ }^{8}$ The signal-to-noise of the instrument in both the DDS and AWG configurations is lower than the original Pate lab spectrometer, ${ }^{8}$ due to the polarization power available (50 W versus $2 \mathrm{~kW}$ ), but comparable to other low power ( $<100 \mathrm{~W})$ CP-FTMW spectrometers. ${ }^{9}, 11,13,23,25$

\section{Full instrument demonstration}

The spectrum of acetone between 9.95 and $13.85 \mathrm{GHz}$ collected using the DDS CP-FTMW is shown in Figure 5. For this demonstration the ADF4158 PLL board was disconnected from the $10 \mathrm{MHz}$ standard for improved performance. ${ }^{26}$ After $22 \mathrm{~h}$ of averaging at a $2.1 \mathrm{kHz}$ repetition rate (170 million averages) the signal-to-noise of the strongest peak was $\sim 18$ 000:1. The dual sideband convolved $(\mathrm{LO}=11900 \mathrm{MHz})$ predicted spectrum of the normal species of acetone ${ }^{27,28}$ and the two ${ }^{13} \mathrm{C}$ substituted species ${ }^{29}$ (scaled to $1 \%$ intensity) at $2 \mathrm{~K}$ are also plotted in Figure 5, with negative intensity. At this sensitivity, the singly substituted ${ }^{13} \mathrm{C}$ isotopomer of acetone is clearly visible in natural abundance at 160:1, as shown in the inset of Figure 5. Additionally, several acetone transitions above $13.9 \mathrm{GHz}$ were aliased into the

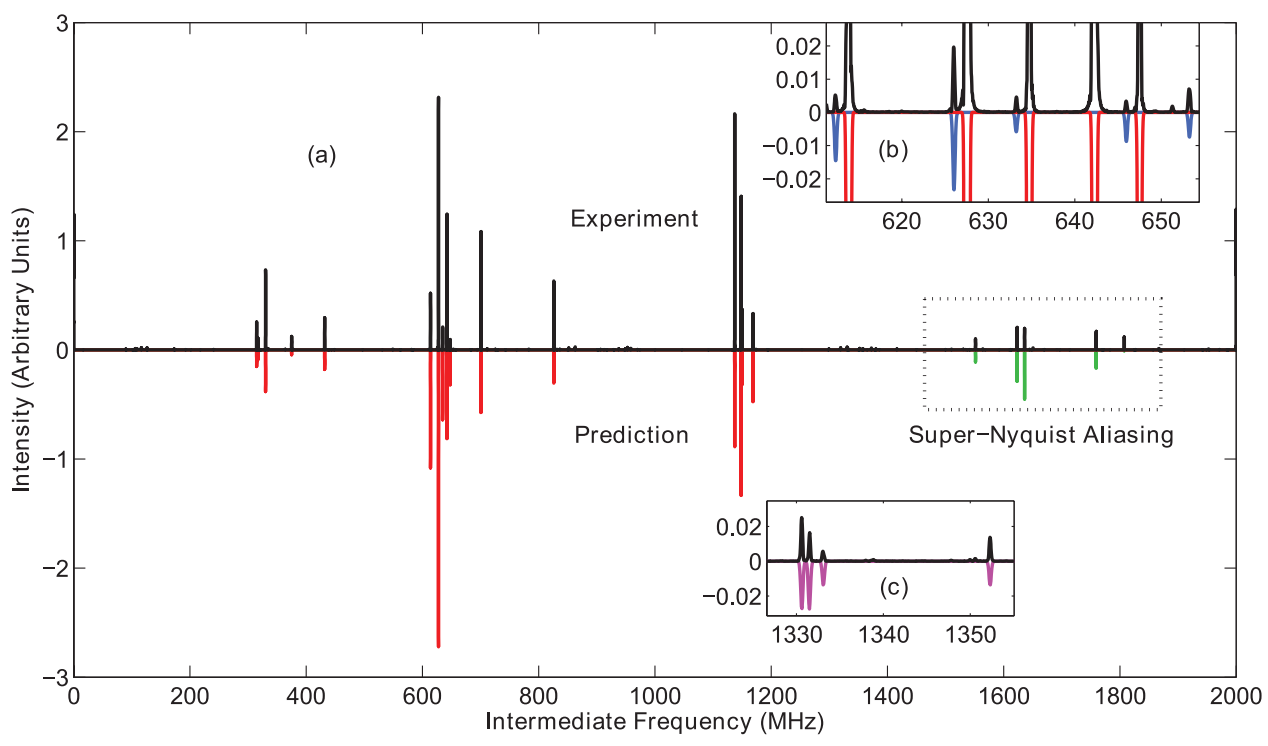

FIG. 5. The dual sideband convolved spectrum of acetone from 9.95 to $13.85 \mathrm{GHz}$ ( $\mathrm{LO}=11.9 \mathrm{GHz}$ ) after $22 \mathrm{~h}$ of averaging (170 million FIDs). (a) The experimental spectrum (positive going) is plotted against a prediction of the normal species (negative intensity). The signal-to-noise is 18000:1 on the strongest transition $\left(2_{11}-2_{02} \mathrm{EE}\right)$. Super-Nyquist content of the normal species at 1.5-1.9 GHz is plotted with a different scaling and highlighted in the dashed box. (b) An inset of the spectrum showing experimental and predicted $2_{11-2}-2$ transitions of the carbonyl ${ }^{13} \mathrm{C}$ substituted species of acetone at $2 \mathrm{~K}$. (c) An inset of the methyl ${ }^{13} \mathrm{C}$ substituted species of acetone at $2 \mathrm{~K}$ showing four of the $3_{21}-3_{12}$ transitions. 

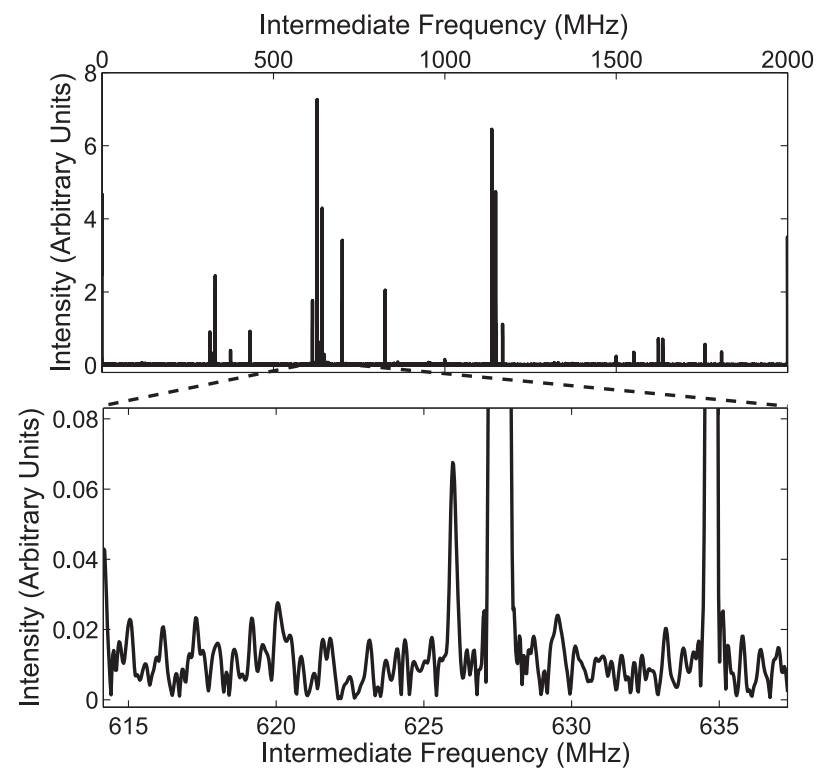

FIG. 6. The spectrum of acetone after $30 \mathrm{~s}$ of data collection (top), with an inset showing the detection of the ${ }^{13} \mathrm{C}$ isotopomer at more than $3: 1$ signal-tonoise (bottom). $\mathrm{LO}=11900 \mathrm{MHz}$.

detection bandwidth. Although the output of the DDS and the input of the digitizer are filtered, a sufficient amount of superNyquist power was delivered from the DDS through the amplifiers to polarize transitions at $2-2.5 \mathrm{GHz}$ offset from the LO frequency. This behavior can be easily removed by using higher rejection low-pass filters. Finally, the rest frequencies of the transitions were verified by shifting the LO frequency.

The relative transition strengths are greatly dependent on the response of the SSA and the coupling of the horn to free space, which causes moderate intensity variations in the measured spectrum. However, the observed relative intensity variations are comparable to those reported in the literature for conventional instruments. ${ }^{8}$

The fast acquisition speed of the DDS CP-FTMW spectrometer is demonstrated in Figure 6. After $30 \mathrm{~s}$ of data collection (65 000 averages) two of the the strongest peaks of the carbonyl substituted ${ }^{13} \mathrm{C}$ isotopomer are visible at 3:1 signalto-noise.

Finally, anomalous doublets were observed for longer (20 $\mu \mathrm{s})$ FID collections. This splitting is present in both the AWG and DDS circuits. The peak splitting increases with helium backing gas, indicating that it is caused by expansion conditions and not by the microwave circuit. However, this splitting does not significantly influence the performance of the spectrometer, since the peak centers can be found to be better than $10 \mathrm{kHz}$ precision.

\section{CONCLUSION}

The utility of the CP-FTMW spectrometer for sensitive detection and characterization of gases with a permanent dipole is well established among molecular spectroscopists. With the latest bandwidth extensions in commercially available DDS chips it is now possible to generate a chirped pulse and LO signal at $12 \mathrm{GHz}$ using an energy-efficient and compact design. Additionally, PLL synthesizers can be expected to increase in frequency (for example, the new $18 \mathrm{GHz}$ Analog Devices ADF41020), and with sufficient multiplication the LO signal could also be supplied by the DDS. Indeed, the inherent step nature of the DDS frequency sweep matches the signal requirements for "stepped" LO CP-FT mm-wave spectroscopy. ${ }^{22,30}$ Multiplication of a DDS chirped pulse has already been demonstrated in a hybrid DDS/PLL chirped pulse synthesizer for radar applications at $675 \mathrm{GHz}^{19}$ and could be used to further extend the bandwidth of the instrument.

Regardless of the specific implementation, the phase stability and harmonic distortion of the DDS chirped pulses are comparable with those of the AWG for short and long averaging times, providing the required stability for coherent signal averaging. Similar advances can be expected in other areas of CP-FTMW technology as well. The complexity of FPGAs are increasing on even faster slopes than $\mathrm{CPU}$ architectures, and high duty cycle digitizers and averagers can, therefore, be expected to continue improving in capabilities well into multi$\mathrm{GHz}$ bandwidth designs. For microwave studies in molecular beams where quasi-optical focusing is required, high power, high cost amplifiers will be needed, but for waveguide studies ${ }^{10}$ or especially higher frequency applications ${ }^{30}$ the amplifier power requirements for sample polarization drop considerably. The low cost of this new DDS-circuit design combined with these advances should allow for the widespread use of CP-FTMW spectroscopy in physical chemistry laboratories, and the capabilities outlined here open the door to truly integrated DDS/PLL single card approaches to compact, energy efficient, lightweight CP-FTMW spectrometers for in situ and process monitoring applications.

\section{ACKNOWLEDGMENTS}

The authors would like to acknowledge the National Science Foundation (Grant No. CHE-1214123 and the Graduate Research Fellowship Program) and National Aeronautics and Space Administration (Grant No. NNX09AM84G) for financial support, the Pate lab for helpful comments and the use of their AWG, Steve Shipman for CP-FTMW advice, Jeff Groseth for electrical support, Bob Dengler for DDS advice, an anonymous referee for a careful and observant review and bringing our attention to the early history of pulsed microwave spectroscopy, and the rest of the Blake lab, especially Brett McGuire, for their support.

${ }^{1}$ A. L. Steber, J. L. Neill, D. P. Zaleski, B. H. Pate, A. Lesarri, R. G. Bird, V. Vaquero-Vara, and D. W. Pratt, Faraday Discuss. 150, 227 (2011).

${ }^{2}$ B. C. Dian, G. G. Brown, K. O. Douglass, and B. H. Pate, Science 320, 924 (2008).

${ }^{3}$ D. Patterson, M. Schnell, and J. M. Doyle, Nature (London) 497, 475 (2013).

${ }^{4}$ E. Herbst and E. F. van Dishoeck, Annu. Rev. Astron. Astrophys. 47, 427 (2009).

${ }^{5}$ R. Dicke and R. Romer, Rev. Sci. Instrum. 26, 915 (1955).

${ }^{6}$ J. Ekkers and W. Flygare, Rev. Sci. Instrum. 47, 448 (1976).

${ }^{7}$ T. Balle and W. Flygare, Rev. Sci. Instrum. 52, 33 (1981).

${ }^{8}$ G. G. Brown, B. C. Dian, K. O. Douglass, S. M. Geyer, S. T. Shipman, and B. H. Pate, Rev. Sci. Instrum. 79, 053103 (2008).

${ }^{9}$ D. A. Obenchain, A. A. Elliott, A. L. Steber, R. A. Peebles, S. A. Peebles, C. J. Wurrey, and G. A. Guirgis, J. Mol. Spectrosc. 261, 35 (2010). 
${ }^{10}$ B. Reinhold, I. A. Finneran, and S. T. Shipman, J. Mol. Spectrosc. 270, 89 (2011).

${ }^{11}$ G. S. Grubbs, C. T. Dewberry, K. C. Etchison, K. E. Kerr, and S. A. Cooke, Rev. Sci. Instrum. 78, 096106 (2007).

${ }^{12}$ A. J. Shirar, D. S. Wilcox, K. M. Hotopp, G. L. Storck, I. Kleiner, and B. C. Dian, J. Phys. Chem. A 114, 12187 (2010).

${ }^{13}$ M. D. Marshall, H. O. Leung, B. Q. Scheetz, J. E. Thaler, and J. S. Muenter, J. Mol. Spectrosc. 266, 37 (2011).

${ }^{14}$ J. Thomas, J. Yiu, J. Rebling, W. Jaeger, and Y. Xu, "Chirped-Pulse and Cavity-Based Fourier Transform Microwave Spectroscopy of a Chiral Epoxy Ester: Methyl Glycidate,” J. Phys. Chem. A (published online).

${ }^{15}$ S. L. Stephens and N. R. Walker, J. Mol. Spectrosc. 263, 27 (2010).

${ }^{16} \mathrm{~S}$. Mata, I. Peña, C. Cabezas, J. López, and J. Alonso, J. Mol. Spectrosc. 280, 91 (2012).

${ }^{17}$ D. S. Wilcox, K. M. Hotopp, and B. C. Dian, J. Phys. Chem. A 115, 8895 (2011).

${ }^{18}$ Analog Devices, “AD9914 Data Sheet,” (Analog Devices, Inc., 2013), http://www.analog.com/static/imported-files/data_sheets/AD9914.pdf.

${ }^{19}$ K. B. Cooper, R. J. Dengler, N. Llombart, B. Thomas, G. Chattopadhyay, and P. H. Siegel, IEEE Trans. Terahertz Sci. Technol. 1, 169 (2011).

${ }^{20} \mathrm{E}$. Alekseev, R. Motiyenko, and L. Margulès, in 65th International Symposium on Molecular Spectroscopy, 2010.

${ }^{21}$ D. B. Holland, I. A. Finneran, P. B. Carroll, and G. A. Blake, "A reducedcost chirped pulse Fourier transform microwave spectrometer using direct digital synthesis," Invention Disclosure Report, provisional patent (4 June 2013).

${ }^{22}$ See supplementary material at http://dx.doi.org/10.1063/1.4818137 for details regarding super-Nyquist operation, stepped-LO operation, and AD9914 implementation.

${ }^{23}$ R. G. Bird, J. L. Neill, V. J. Alstadt, J. W. Young, B. H. Pate, and D. W. Pratt, The J. Phys. Chem. A 115, 9392 (2011).

${ }^{24}$ I. Peña, A. M. Daly, C. Cabezas, S. Mata, C. Bermúdez, A. Niño, J. C. Lopez, J.-U. Grabow, and J. L. Alonso, J. Phys. Chem. Lett. 4, 65 (2013).

${ }^{25}$ M. K. Jahn, D. A. Dewald, D. Wachsmuth, J.-U. Grabow, and S. C. Mehrotra, J. Mol. Spectrosc. 280, 54 (2012).

${ }^{26} \mathrm{We}$ observed less frequency jitter in the ADF4158 when using the internal reference on the evaluation board. However, while recording these data we realized that the internal clock on the PLL board was not properly isolated from the external clock input. After removing the resistors connecting the internal clock to the PLL, and connecting the board to the external $\mathrm{Rb}$ standard, we observed optimal performance.

${ }^{27}$ P. Groner, S. Albert, E. Herbst, F. C. De Lucia, F. J. Lovas, B. J. Drouin, and J. C. Pearson, Astrophys. J., Suppl. Ser. 142, 145 (2002).

${ }^{28}$ J. Swalen and C. Costain, J. Chem. Phys. 31, 1562 (1959).

${ }^{29}$ F. Lovas and P. Groner, J. Mol. Spectrosc. 236, 173 (2006).

${ }^{30}$ A. L. Steber, B. J. Harris, J. L. Neill, and B. H. Pate, J. Mol. Spectrosc. 280, 3 (2012). 